\title{
The trials and tribulations of guinea pig pros
}

The Professional Guinea Pig: Big Pharma and the Risky World of Human Subjects Roberto Abadie

Duke University Press; 2010.

$\mathrm{W}$ hen referring to patients, it is hard to find a term more offensive than "guinea pig." The connotations are the furthest possible from evidence-based medicine, the primacy of the patients' needs and wishes, the importance of informed consent and the duty to do no harm. Yet there are enthusiastic cohorts of healthy people who don't mind the label and are eager to devote their bodies to medical science for money. Today's selfproclaimed professional guinea pigs are the thousands of healthy volunteers who are enrolled in Phase I trials to determine the metabolic and pharmacologic actions of drugs in humans, as well as the side effects associated with various doses. Initial testing of drugs in humans has always been a somewhat ethically murky endeavour. Formerly restricted to prisoners, the practice has shifted to urban subcultures where the unemployed, students and other low-income earners gravitate to it as an easy - yet often challenging - way to make some money.

Author and anthropologist Roberto Abadie penetrates the professional corps of human test subjects in his book The Professional Guinea Pig: Big Pharma and the Risky World of Human Subjects to uncover often shocking accounts of the lives of professional and semiprofessional test subjects. Abadie is no casual observer, either. As a medical ethnographer, he spent over a year living in Philadelphia, Pennsylvania, home to much pharmaceutical research, immersed in guinea pig culture, interviewing and meticulously documenting the experiences of a handful of volunteer human test subjects.

It is a treat to read an observant and thoughtful account by someone who has

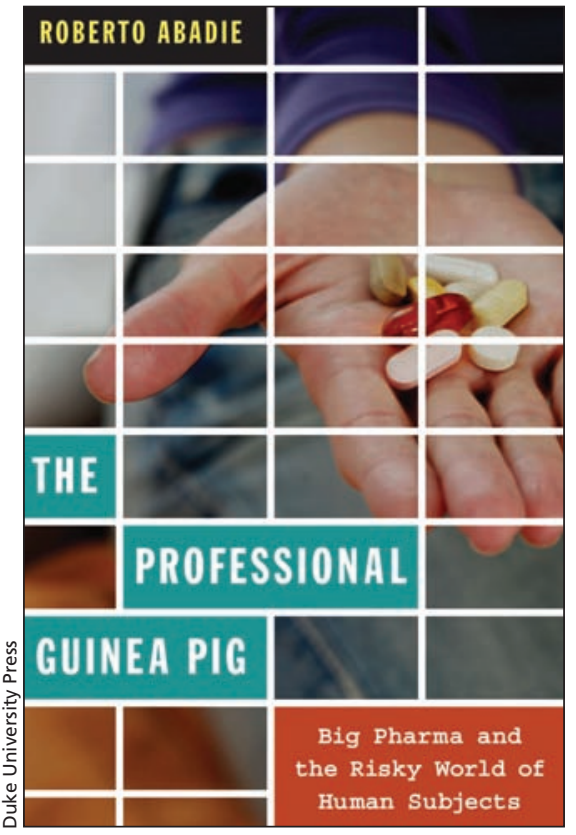

escaped the traditional halls of academia, to delve into the world inhabited by the test subjects at the centre of his research. He asks fundamental questions: Who are these people? Where and how do they live? What drives them to subject themselves to the potential harm, humiliation and crushing boredom of clinical trials? And perhaps the most disturbing question of all: Can we trust the information that comes out of those trials?

Readers will learn some surprising things in this book, such as the degree to which professional guinea pigs as well as those who run clinical trials "game" the system, which is understandably loaded with rules and procedures. Those on both sides of the trial - those who need the subjects and those who need the money - do an elaborate dance around rules which are designed to protect the safety of the volunteer and the integrity of the data. An unspoken collusion permeates the relationships between the contract research organizations hired by the drug companies to conduct studies and the pliable cohort of professional test subjects, without whom no drug would get approved.
One guinea-pigging patient, Spam, describes the trials as a "mild torture economy" where one is not actually paid to do anything except "endure." The Phase I patient has nothing to benefit, but money and so getting enrolled (where sometimes blatant lies have to be told) and staying in a trial (to collect the bonus for completing it) are the key requirements of the job.

Roberto Abadie maps out the underlying tension that Phase I trials construct. With the obvious imbalance between the poor or unemployed who offer their bodies for money and the contract research organization which can fork over $\$ 3000$ for a patient's body for a three-week trial, the financial incentives on both sides of the clinical trial will always be based on a sketchy form of informed consent. If guinea pigs give their bodies or rent them to the mild torture economy, can they be fully informed, without coercion or force before doing so?

That's the most challenging question this book attempts to answer. Unfortunately, so much of our medical system depends on the services of professional guinea pigs - both the paid ones, as Abadie's fine book demonstrates, as well the rest of us, the unpaid ones who in swallowing a new drug will always be part of an adventure that's not fully known.

The goal, then in both research and policy should not be to cynically believe that we're all helpless rodents, but that we are thinking beings able to more comprehensively navigate an ethically challenging terrain, so that both parties, drug companies and the patients they depend on to study their drugs, will ultimately benefit from the experience.

\section{Alan Cassels MPA \\ Drug policy researcher \\ University of Victoria \\ Victoria, BC}

CMAJ 2011. DOI:10.1503/cmaj.101829 